ARTICLE

Received 5 May 2014 | Accepted 17 Jun 2014 | Published 16 Jul 2014

DOI: $10.1038 /$ ncomms5442 OPEN

\title{
Rewiring neural circuits by the insertion of ectopic electrical synapses in transgenic $C$. elegans
}

Ithai Rabinowitch ${ }^{1,2, \star}$, Marios Chatzigeorgiou ${ }^{1, \star}$, Buyun Zhao ${ }^{1}$, Millet Treinin ${ }^{2} \&$ William R. Schafer ${ }^{1}$

Neural circuits are functional ensembles of neurons that are selectively interconnected by chemical or electrical synapses. Here we describe a synthetic biology approach to the study of neural circuits, whereby new electrical synapses can be introduced in novel sites in the neuronal circuitry to reprogram behaviour. We added electrical synapses composed of the vertebrate gap junction protein $\mathrm{C} \times 36$ between Caenorhabditis elegans chemosensory neurons with opposite intrinsic responses to salt. Connecting these neurons by an ectopic electrical synapse led to a loss of lateral asymmetry and altered chemotaxis behaviour. In a second example, introducing $\mathrm{C} \times 36$ into an inhibitory chemical synapse between an olfactory receptor neuron and an interneuron changed the sign of the connection from negative to positive, and abolished the animal's behavioural response to benzaldehyde. These data demonstrate a synthetic strategy to rewire behavioural circuits by engineering synaptic connectivity in C. elegans.

\footnotetext{
${ }^{1}$ Division of Cell Biology, MRC Laboratory of Molecular Biology, Francis Crick Avenue, Cambridge CB2 OQH, UK. ${ }^{2}$ Department of Medical Neurobiology, Hadassah Medical School, Hebrew University of Jerusalem, Jerusalem 9112102, Israel. ${ }^{*}$ These authors contributed equally to this work. Correspondence and requests for materials should be addressed to W.R.S. (email: wschafer@mrc-Imb.cam.ac.uk).
} 
central aim of neuroscience research is to reveal the function of neural circuits and how changes in synaptic connectivity modify behaviour. Recent advances in synthetic biology, whereby genetic networks of living cells have been rewired to produce alternative cellular function ${ }^{1-3}$, may revolutionize the field of cell biology. Synthetic approaches for re-engineering neural function and behaviour have also begun to be developed for neuroscience; in particular, optogenetic and other protein-based reagents have been used to manipulate the activity patterns of defined neurons in vivo ${ }^{4,5}$. In principle, a synthetic neurobiology approach could also be used to manipulate neural circuits by artificially modifying specific synaptic connections in the circuit and thus reprogramming behaviour.

To artificially modify the connection between neurons we sought to introduce a new synapse into an existing neural circuit. Inserting heterologous chemical synapses into a circuit would be difficult owing to their enormous complexity and hundreds of constituent proteins ${ }^{6,7}$. In contrast, electrical synapses, or gap junctions, consist of as little as one protein type ${ }^{8}$, and it has been shown that overexpression of an endogenous gap junction protein can alter neuronal connectivity in vitro ${ }^{9}$. The molecular constituents of vertebrate and invertebrate gap junctions belong to distinct protein families: connexins are exclusive to vertebrates and innexins to invertebrates ${ }^{10-12}$. Importantly, while proteins from the same family can interact to form heterotypic gap junctions ${ }^{13,14}$, no compatibility has been found between connexins and innexins ${ }^{15}$. Thus, we reasoned that connexins expressed heterologously in Caenorhabditis elegans neurons would form gap junctions exclusively between themselves, and that these new connections could be used to introduce novel connections between normally unconnected neurons in intact animals. Here we present data assessing the use of this approach in two neural circuits, involved in salt taste and olfactory chemotaxis.

\section{Results}

Adding synthetic electrical synapses between sensory neurons. We first investigated the possibility of rewiring neuronal connections in vivo using the neural circuit involved in salt chemotaxis. C. elegans navigates up gradients of sodium chloride and other salts in part through a biased random walk ${ }^{16}$, combining forward travel with stochastically occurring reorienting turnings and reversals. The primary neurons sensing attractive concentrations of salt are the bilaterally symmetric ASE neurons ${ }^{17}$; ASEL is an ON cell that is stimulated by an increase in salt concentration, while ASER is an OFF cell stimulated by a salt concentration decrease ${ }^{18}$. Unlike most bilateral sensory neuron pairs in C. elegans, the ASEL and ASER neurons are not normally connected by gap junctions (Fig. 1a). Published connectome data likewise report no chemical synapses between ASEL and ASER ${ }^{19,20}$, although more recent online data based on computer-aided reconstructions ${ }^{21,22}$ suggest some may exist. Moreover, confocal imaging of ASER and ASEL, labelled with expressed fluorescent proteins of different colours, showed that the processes of these two neurons are physically adjacent within the nerve ring (Fig. 1b).

We therefore reasoned that heterologous connexin expression could be used to synthetically connect the ASEL and ASER neurons with electrical synapses. We generated a synthetic connexinderived transgene consisting of a C. elegans codon-optimized ${ }^{23}$, fluorescently tagged version of the mouse connexin 36 (Cx36), a well-characterized, broadly expressed gap junction constituent ${ }^{24}$, under the control of promoters specific for either ASER $(g c y-5)$ or ASEL $(g c y-7)$. In multicopy extrachromosomal lines overexpressing Cx36 in both neurons $\left(\mathrm{ASER}_{\mathrm{C} \times 36} \mathrm{ASEL}_{\mathrm{Cx} 36}\right.$ ), as well as a line expressing $\mathrm{Cx} 36$ in ASER alone $\left(\mathrm{ASER}_{\mathrm{Cx} 36}\right)$, this tagged $\mathrm{Cx} 36$ protein localized throughout the dendrite, axon and cell body in a punctate pattern (Fig. 1c). In some but not all cases, $A_{S E R}{ }_{\mathrm{Cx} 36}$ puncta were coincident to $\mathrm{ASEL}_{\mathrm{Cx} 36}$ puncta, suggesting that they might represent synthetic gap junctions (Supplementary Fig. 1).

To test whether Cx36 expression could indeed generate ectopic gap junctions linking ASEL and ASER, we performed calciumimaging experiments to investigate the effects of $A_{S E R}{ }_{C \times 36}$ and $\mathrm{ASEL}_{\mathrm{Cx} 36}$ on neuronal responses to chemosensory stimulation. Although genetically encoded calcium indicators lack the temporal sensitivity to detect rapid electrical potentials, they are well-suited to detect changes in the slow, graded responses to $\mathrm{NaCl}$ expected from the introduction of electrical coupling between ASEL and ASER. In wild-type animals, a step change from 40 to $0 \mathrm{mM} \mathrm{NaCl}$ evoked a calcium increase in ASER but no response in ASEL (Fig. 1d, blue). Expressing Cx36 in ASEL or ASER alone did not significantly alter either response (Fig. 1f,g). In contrast, in ASER $_{\mathrm{Cx} 36} \mathrm{ASEL}_{\mathrm{Cx} 36}$ animals both ASEL and ASER showed robust calcium increases in response to the downstep stimulus (Fig. 1d, red), as predicted if the cells were electrically coupled. We also assayed the responses of Cx36-expressing strains to increases in $\mathrm{NaCl}$ concentration. We observed that in wild-type, $\mathrm{ASEL}_{\mathrm{Cx} 36}$ and $\mathrm{ASER}_{\mathrm{Cx} 36}$ animals, salt upsteps led to a calcium increase in ASEL and a calcium decrease in ASER (Fig. 1e,h,i). However, in $\mathrm{ASER}_{\mathrm{Cx} 36} \mathrm{ASEL}_{\mathrm{Cx} 36}$ animals (Fig. 1e, red), ASER showed a calcium increase in response to a salt upstep, as expected if the depolarizing current in ASEL depolarized ASER through an electrical connetion. These alterations in ASE activity correlated with a significant defect in the ability of the animals to navigate in a point-source gradient of $\mathrm{NaCl}$ (Supplementary Fig. 2a). Together, these results indicate that functional electrical synapses were successfully inserted between ASEL and ASER.

Flipping the sign of an inhibitory synapse. We next tested whether existing neuronal connections could be reconfigured through ectopic Cx36 expression. To do this we focused on the AWC and AIY neurons, which mediate chemotaxis towards attractive odourants, such as benzaldehyde ${ }^{25,26}$. The AWC sensory neurons respond to increases in odour concentration with reduced activity and to decreases in odour concentration with elevated activity. The AWCs make inhibitory chemical synapses with the AIY interneurons (Fig. 2a); although AIY ablation does not impair chemotaxis to odourants ${ }^{27}$, optogenetic AIY activation has been shown to promote forward travel and inhibit reorientations ${ }^{28}$. To determine whether heterologous connexin expression could modify the properties of these synapses and thereby impact behaviour, we expressed Cx36 in AWC and AIY using promoters derived from odr-1 (for $\mathrm{AWC}_{\mathrm{Cx} 36}$ ) and the $t$ tx-3 second intron (for $\mathrm{AIY}_{\mathrm{Cx} 36}$ ) and imaged calcium responses to olfactory stimuli using genetically encoded calcium indicators (Fig. 2b,c; Supplementary Fig. 3). In wild-type animals, benzaldehyde presentation led to a calcium decrease in AWC and a reciprocal calcium increase in AIY, as expected from their known inhibitory connection (Fig. 2d). In animals expressing either the $\mathrm{AWC}_{\mathrm{Cx} 36}$ or the $\mathrm{AIY}_{\mathrm{Cx} 36}$ transgenes alone, these responses were not significantly altered (Fig. 2f,g). In contrast, animals expressing $\mathrm{Cx} 36$ in both neurons $\left(\mathrm{AWC}_{\mathrm{Cx} 36} \mathrm{AIY}_{\mathrm{Cx} 36}\right)$ responded to benzaldehyde presentation with a calcium decrease in both AWC and AIY, suggesting that ectopic gap junctions between AWC and AIY reversed the sign of the AWC-AIY connection from negative to positive (Fig. 2d, red). A similar effect was observed when the $\mathrm{AWC}_{\mathrm{Cx} 36} \mathrm{AIY}_{\mathrm{Cx} 36}$ transgenes were carried on a chromosomally integrated array (Supplementary Fig. 4). We also observed an attenuation in the degree of $\mathrm{AWC}$ hyperpolarization in the $\mathrm{AWC}_{\mathrm{Cx} 36} \mathrm{AIY}_{\mathrm{Cx} 36}$ 
a

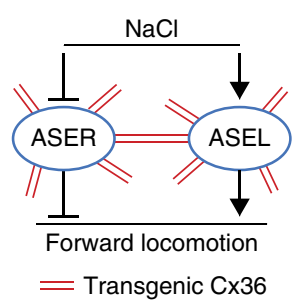

b

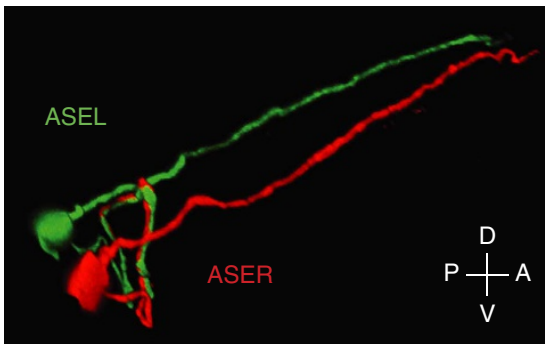

C

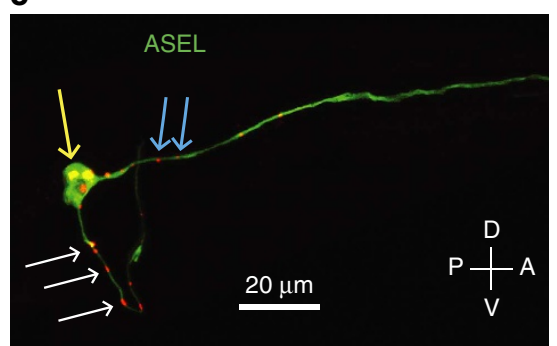

d

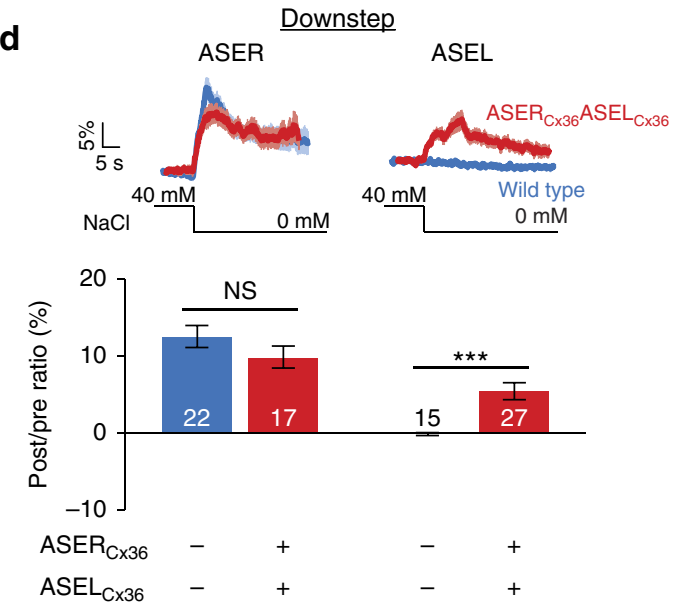

Downstep

f
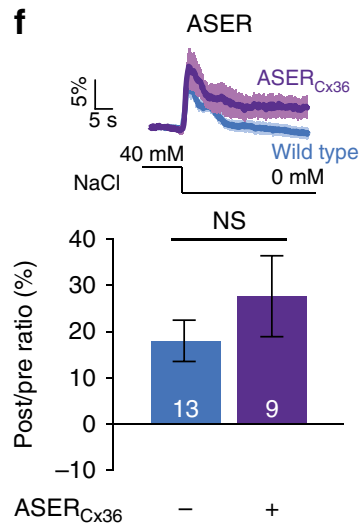

g
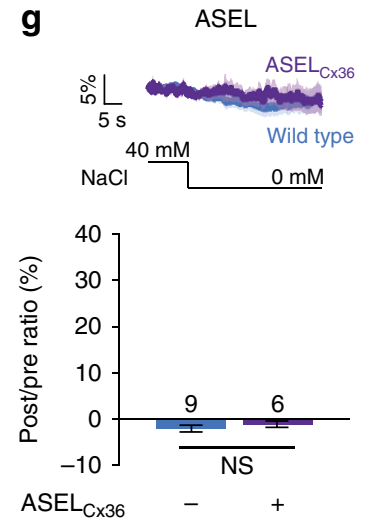

e

Upstep
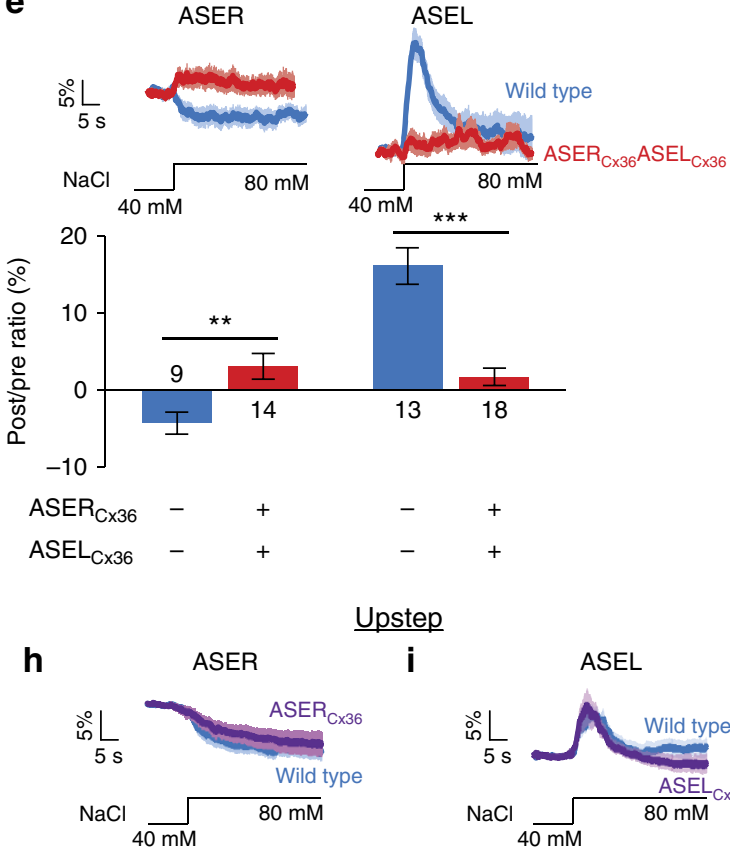

Upstep

i
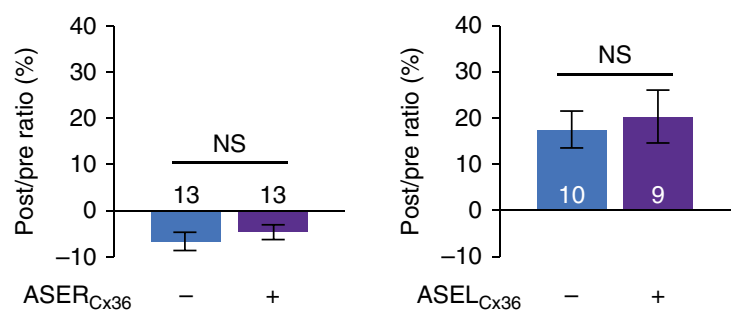

Figure 1 | An engineered electrical synaptic connection functionally couples ASER and ASEL. (a) ASEL and ASER participate in a circuit for salt sensation and do not have any natural electrical connections. Chemical synapses between ASEL and ASER may be present in wild-type animals ${ }^{22}$, although the loss of synaptic transmission is not reported to affect ASEL and ASER sensory responses ${ }^{18}$. (b) The axons of ASER (red) and ASEL (green) appear to contact each other, as revealed by confocal microscopy. Shown is a three-dimensional confocal image of strain AQ3309 (gcy-7::gfp, gcy-5::mCherry; see strain list). (c) Confocal image of strain AQ3311, in which ASEL (green) expresses the genetically encoded calcium indicator YC3.60 and Cx36 tagged with mCherry (red). Cx36 puncta in the cell body (yellow arrow), dendrite (blue arrows) and axon (white arrows) are indicated. Scale bar, $20 \mu \mathrm{m}$. (d,e) Calcium imaging of ASER and ASEL responses to a downstep (d) or upstep (e) in $\mathrm{NaCl}$ concentration in wild-type worms and in worms expressing Cx36 in both ASER and ASEL. (f-i) Calcium imaging of ASER (f,h) or ASEL (g,i) responses to a downstep (f,g) or upstep (h,i) in NaCl concentration in wild-type worms and in worms expressing Cx36 in ASER (f,h) or ASEL (g,i) alone. At the top of each panel are averaged traces with shaded regions indicating s.e.m.; blue traces indicate wild-type, purple traces single neuron connexin lines, and red traces lines expressing Cx36 in both ASEL and ASER. At the bottom of each panel are percent mean fluorescent ratios, $10 \mathrm{~s}$ after compared with $10 \mathrm{~s}$ before stimulus onset. Two-tailed unpaired $t$-tests, ${ }^{\star \star} P<0.01,{ }^{\star \star \star} P<0.001, N S$, not significant. Error bars represent s.e.m. Numbers on bars indicate sample sizes.

worms, consistent with a shunting of the hyperpolarizing current to AIY (Fig. 2d, red). Together these results indicate that ectopic gap junctions were formed between AWC and AIY, switching an inhibitory chemical synapse to a predomininantly excitatory electrical/chemical synapse.

We also analysed the effect of ectopic connexin expression on responses to benzaldehyde removal. As expected, in wild-type worms this led to a calcium increase in AWC and a small calcium decrease in AIY, consistent with an inhibitory chemical synapse (Fig. 2e). In contrast, $\mathrm{AWC}_{\mathrm{Cx} 36} \mathrm{AIY}_{\mathrm{Cx} 36}$ worms showed a calcium increase in AIY, an attenuated calcium increase in AWC (Fig. 2e, red) and a strong defect in benzaldehyde chemotaxis (Supplementary Fig. 2b), consistent with the presence of an ectopic electrical connection. We also assayed $\mathrm{AWC}_{\mathrm{Cx} 36}$ and 
a

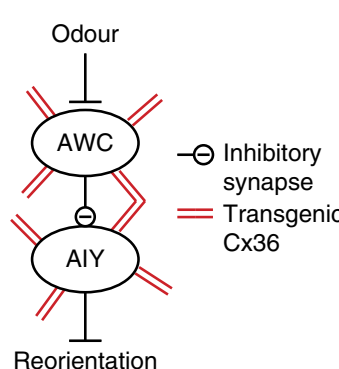

b

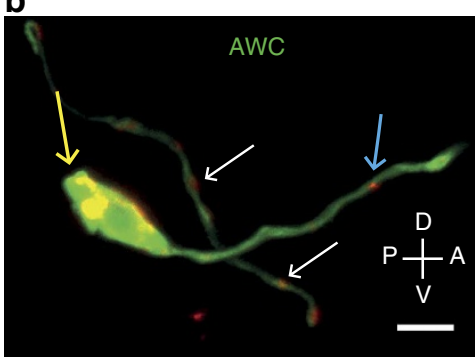

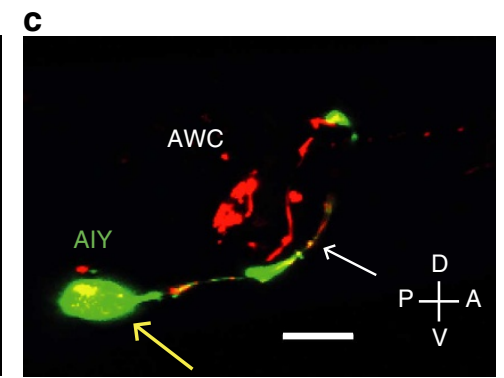

Upstep

d

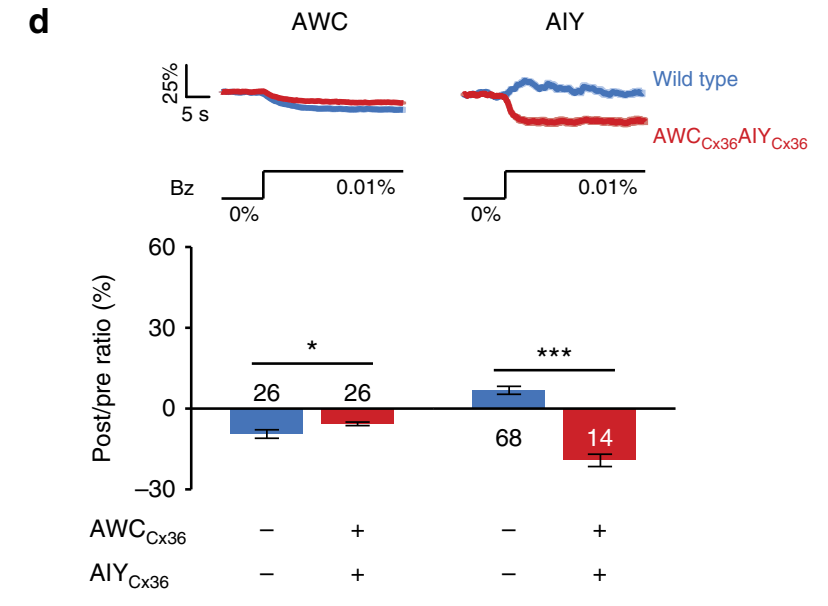

Upstep

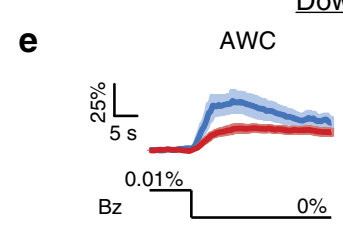

Downstep

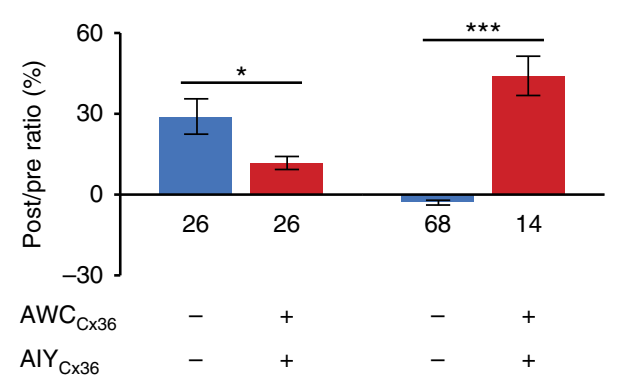

$\mathbf{f}$

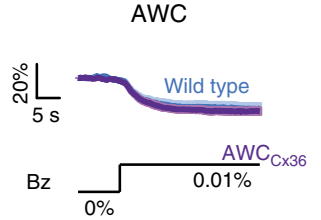

g
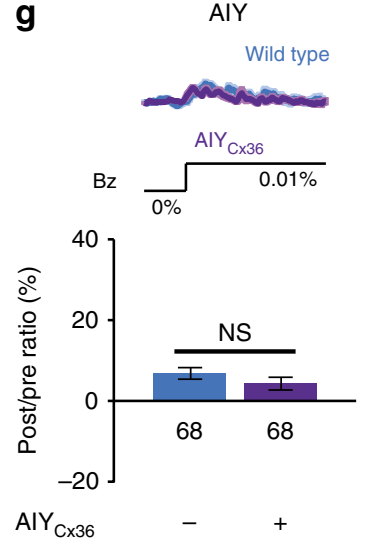

h

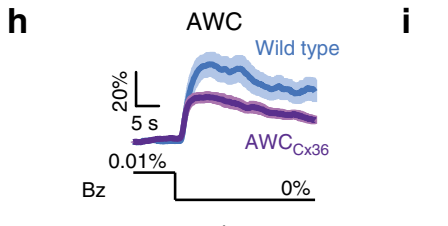

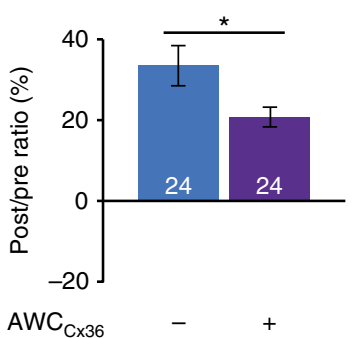

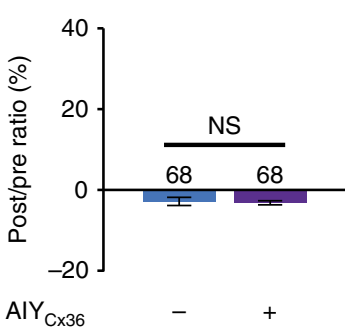

Figure 2 | An engineered electrical synaptic connection between AWC and AIY flips the AIY benzaldehyde response profile. (a) AWC and AIY participate in a circuit for chemosensation and are naturally connected by an inhibitory chemical synapse. (b) Confocal projection image of strain AQ2660, in which AWC (green) expresses the calcium indicator YC3.60, and mCherry-tagged Cx36 puncta (red) are seen in the cell body (yellow arrow), dendrite (blue arrow) and axon (white arrows). Scale bar, $5 \mu \mathrm{m}$. (c) Confocal image of strain transgenic line AQ2646, in which mCherry-tagged Cx36 is expressed in AIY and AWC (red punctate fluorescence), and YC3.60 is expressed in AIY (green fluorescence). Yellow and white arrows indicate the cell body and axon respectively. Scale bar, $10 \mu \mathrm{m}$. An expanded version of this image and version showing the red channel alone are in Supplementary Fig. 3. (d,e) Calcium imaging of AWC and AIY responses to an upstep (d) or downstep (e) in benzaldehyde (Bz) concentration in wild-type worms and in worms expressing Cx36 in both AWC and AIY. (f-i) Calcium imaging of AWC (f,h) or AIY (g,i) responses to an upstep (f,g) or downstep (h,i) in Bz concentration in wild-type worms and in worms expressing Cx36 in AWC $(\mathbf{f}, \mathbf{h})$ or AIY $(\mathbf{g}, \mathbf{i})$ alone. At the top of each panel are averaged traces with shaded regions indicating s.e.m. At the bottom of each panel are percent mean fluorescent ratios, $10 \mathrm{~s}$ after compared with $10 \mathrm{~s}$ before stimulus onset. Two-tailed unpaired $t$-tests, ${ }^{\star} P<0.05$, ${ }^{\star \star \star} P<0.001$, NS, not significant. Error bars represent s.e.m. Numbers on bars indicate sample sizes.

$\mathrm{AIY}_{\mathrm{Cx} 36}$ strains individually. We observed that $\mathrm{AIY}_{\mathrm{Cx} 36}$ worms showed normal responses to benzaldehyde removal and normal benzaldehyde chemotaxis, whereas $\mathrm{AWC}_{\mathrm{Cx} 36}$ worms showed reduced calcium influx and a partial deficiency in chemotaxis (Fig. 2h,i; Supplementary Fig. 2b). Since the left and right AWCs, unlike most amphid neurons, are not normally linked by gap junctions, we reasoned that $\mathrm{AWC}_{\mathrm{Cx} 36}$ expression might alter the neurons' electrical properties by electrically coupling the AWC pair. Consistent with this possibility, when we expressed Cx36 in only one of the two AWCs, (AWC ${ }^{\text {off }}$ ) using the $\operatorname{srs} x-3$ promoter $^{29}$, we observed wild-type calcium responses and normal chemotaxis (Supplementary Fig. 5), consistent with the possibility that the attenuation in $\mathrm{AWC}_{\mathrm{Cx} 36}$ activity was due to the formation of a new electrical connection between AWCR and 
AWCL. Alternatively, although our odr-1 promoter fragment appears AWC-specific in our lines, if the odr-1::Cx36 transgene is driving low-level Cx36 expression in other neurons, these might form ectopic gap junctions with the AWCs and thereby attenuate AWC responses by shunting.

\section{Discussion}

In summary, we have shown using two different neural circuits in C. elegans that vertebrate connexins can be used to create synthetic electrical synapses in an intact nervous system. By transgenically targeting connexin expression to physically adjacent neurons, this manipulation can be used as a general technique for artificially modifying neural connectivity. This approach complements several existing methods for manipulating the excitability ${ }^{30}$ or efficacy of synaptic transmission ${ }^{31,32}$ from genetically targeted neurons. Ectopic connexin expression makes it possible not only to generate new connections between unconnected neurons, but also to modify the strength of existing electrical synapses ${ }^{33}$. Thus, synthetic modification of neural connections should prove to be a useful tool to obtain insight into the workings of neural circuits in genetically tractable animals, in which the precise connectivity and the relative position of each circuit component is known.

The approach described here takes advantage of the fact that vertebrate and invertebrate gap junctions are formed from distinct, most likely incompatible protein families. Thus, an ectoptic connexin hemichannel is not expected to pair with endogenous innexin hemichannels in C. elegans neurons; indeed, the observation that $\mathrm{AWC}_{\mathrm{C} \times 36}$ does not on its own lead to electrical coupling to AIY (which express the endogenous innexin inx-18 (ref. 34)) supports this conjecture. Although this would appear to limit the exact method described here to invertebrate nervous systems, it has been found that invertebrate innexins can readily be expressed to form hemichannels in vertebrate Xenopus oocytes $^{14}$. It is therefore feasible that a mirror image of the method we describe here (that is, ectopic expression of innexins in vertebrates) might be used to modify the connectivity of mammalian brain circuits. Synthetic synaptic modification approaches of this sort have a broad range of potential applications in neuroscience research, including the implementation of theoretical neural circuit models in real live circuits, the ability to test the sufficiency (as opposed to necessity) of particular connections for neuronal circuit function and even the engineering of novel circuits and behaviours.

\section{Methods}

Strains. Strains were grown and maintained under standard conditions. Wild-type animals were C. elegans Bristol strain N2. Other strains generated in this study were:

AQ2660 ljEx351[odr-1::YC3.60*, elt-2::mCherry]; ljEx355[odr-1::Cx36*: mCherry, unc-122::mCherry].

AQ2632 ljEx351[odr-1::YC3.60*, elt-2::mCherry].

AQ2640 ljEx355[odr-1::Cx36*::mCherry, unc-122::mCherry]

AQ2795 ljEx411[srsx-3::Cx36*::mCherry, unc-122::mCherry].

AQ2817 ljEx413[srsx-3::YC3.60*]

AQ2818 ljEx413[srsx-3::YC3.60*]; ljEx411[srsx-3::Cx36*::mCherry,

unc-122:: $m$ Cherry].

AQ2610 ljEx336[ttx-3(int2)::Cx36*::mCherry, unc-122::gfp].

AQ2614 ljEx339[odr-1::Cx36*::mCherry,ttx-3(int2)::Cx36*::mCherry unc-122:: $m$ Cherry].

AQ2637 ljEx354[ttx-3(int2)::YC3.60*].

AQ2646 ljEx354[ttx-3(int2)::YC3.60*]; ljEx339[odr-1::Cx36*::mCherry

ttx-3(int2)::Cx36*::mCherry, unc-122::mCherry].

AQ2941 ljEx135[flp-6::YC2.12]; ljEx472[gcy-5::Cx36*::mCherry,

gcy-7::Cx36*::mCherry, unc-122::mCherry].

AQ3239 ljEx135[flp-6::YC2.12]; ljEx610[gcy-5::Cx36*::mCherry,

unc-122:: mCherry].

AQ3309 ljEx668[gcy-7::gfp, gcy-5::mCherry, unc-122::mCherry].

AQ3310 ljEx669[gcy-7::Cx36*::yfp, gcy-5::Cx36*::mCherry, unc-122::mCherry].

AQ3311 ljEx670[gcy-7::cx36*::mCherry, gcy-7::YC3.60, unc-122::mCherry].
MF302 mfIs13[odr-1::Cx36*::yfp, ttx-3(int2)::Cx36*::mCherry, punc-122::mCherry].

Visualizing Cx36 expression in transgenic animals. The complementary DNA sequence of Mus musculus gap junction protein, delta 2 (Gjd2) was codon optimized $^{23}$ to produce a synthetic $C \times 36$ gene indicated in the strain list as $C \times 36^{*}$ (GeneArt). We fused to Cx36 an upstream promoter $\left(g c y-5\right.$ for $\mathrm{ASER}^{35}, g c y-7$ for $\mathrm{ASEL}^{35}$, odr-1 for AWC ${ }^{25}, s r s x-3$ for AWC ${ }^{\text {off } 29}$ and the second intron of $t t x-3$ for $\mathrm{AIY}^{36}$ ) and a downstream gene encoding a fluorescent protein (YFP or mCherry). Plasmids were injected at $70 \mathrm{ng} \mathrm{\mu l}^{-1}$ each, together with a coinjection marker (unc$122:: g f p$, unc-122::mCherry or elt-2::mCherry at 40,40 or $20 \mathrm{ng}^{-1} \mathrm{l}^{-1}$, respectively) to generate transgenic worms carrying an extrachromosomal array. To express an electrical synapse linking two neurons, we co-injected plasmids for both neurons in the same worm. Transgenic Cx36 expression was visualized using an LSM 510 or LSM 780 confocal laser scanning microscope (Zeiss), captured and processed by Zen (Zeiss) software and further processed using Volocity, Fiji and Adobe Illustrator CS3. After scanning a stack of $\sim 1-\mu \mathrm{m}$-thick slices, either a maximum projection image or a three-dimensional rendering was generated and uniformly adjusted for brightness and contrast. Strains for imaging the overlap between the ASE neurons were prepared by co-injecting $g c y-7:: g f p$ and $g c y-5:: m$ Cherry at $85 \mathrm{ng}^{-1} \mathrm{l}^{-1}$ each, with $40 \mathrm{ng}^{-1}$ of $u n c-122:$ : $\mathrm{mCherry}$. These images were acquired and processed as mentioned above. For the deconvolution images in Supplementary Fig. 1, the samples were prepared by immobilizing the worms on a $3 \%$ pad of agarose using $200 \mathrm{mM}$ of sodium azide. The worms were imaged using API Deltavision with a $100 \times$ objective and processed using PerkinElmer Volocity.

Chemotaxis assays. Salt chemotaxis assays were performed essentially as described $^{37}$. Briefly, we poured $10 \mathrm{ml}$ of buffered agar $(2 \%$ agar, $1 \mathrm{mM} \mathrm{CaCl} 2$, $1 \mathrm{mM}$ MgSO4, $5 \mathrm{mM}$ KPO4) into 10-cm diameter Petri dishes. To form the gradient, $16 \mathrm{~h}$ before the assay we applied $10 \mu \mathrm{l}$ of $250 \mathrm{mM} \mathrm{NaCl}$ salt solution adjusted to $\mathrm{pH} 6.0$ to the attractant spot and $10 \mu \mathrm{l}$ of double-distilled $\mathrm{H}_{2} \mathrm{O}$ to the control spot. Four hours before the assay, we added another $4 \mu \mathrm{l}$ of salt solution or double-distilled $\mathrm{H}_{2} \mathrm{O}$ to the same spots. Ten minutes before the assay, we applied $1 \mu \mathrm{l}$ of $1 \mathrm{M}$ sodium azide to both attractant and control spots to immobilize the worms once they reached these spots. We used synchronized animals that were grown on 6-cm nematode growth media plates with food, washed three times with CTX solution ( $1 \mathrm{mM} \mathrm{CaCl} 2,1 \mathrm{mM} \mathrm{MgSO} 4,5 \mathrm{mM}$ KPO4) and pipetted onto a nylon membrane to dry. Subsequently $\sim 200$ worms were placed in the center of the assay plate inside a circle of diameter of $1.5 \mathrm{~cm}$. We allowed the animals to move about the agar surface for $1 \mathrm{~h}$. The chemotaxis index was calculated as the number of animals contained in a circle of radius $1.5 \mathrm{~cm}$ around the salt spot minus the number of worms in a circle of radius $1.5 \mathrm{~cm}$ around the control spot, divided by the total number of animals in the two circles.

Benzaldehyde chemotaxis assays were performed as described ${ }^{38}$, except that they were performed in smaller $6-\mathrm{cm}$ plates, and with $N=20$ worms, which were picked and transferred to the assay plate after being released from food on an empty plate. A drop of $1 \%$ benzaldehyde in ethanol and ethanol alone were placed at two opposite ends of the assay plate together with $1 \mathrm{mM}$ sodium azide for trapping the worms. Chemotaxis was scored the following day by counting the number of paralyzed worms near the odour spot $N$ (odour) and the number of paralyzed worms near the control spot $N$ (control), and calculating the chemotaxis index ${ }^{38}$ equal to: $(N($ odour $)-N($ control $)) /(N($ odour $)+N($ control $))$.

Calcium imaging. Imaging was performed on a Zeiss Axioscope upright microscope using an Andor iXon EM camera or a Hamamatsu ORCA-ER camera in combination with neutral density filters to reduce the intensity of ultraviolet illumination. Movies were captured by IQ1.9 software (Andor) or a custom-written Matlab program and analysed using another custom-written Matlab (Mathworks) script. A rectangular region of interest (ROI) was drawn surrounding the cell body (AWC and ASE) or the neurite (AIY), and for every frame the ROI was shifted according to the new position of the center of mass (in the case of the cell body) or point of maximum intensity (for the AIY neural processes). The fluorescence intensity, $F$, was computed as the difference between the sum of pixel intensities and the faintest $10 \%$ pixels (background) within the ROI. For ratiometric imaging, $\mathrm{ROI}_{\mathrm{Y}}$ tracked the neuron in the yellow channel, and in the cyan channel, $\mathrm{ROI}_{\mathrm{C}}$ moved at a fixed offset from $\mathrm{ROI}_{\mathrm{Y}} . F$ was computed as $F_{\mathrm{Y}} / F_{\mathrm{C}}$ after correcting for bleed through ${ }^{37}$. No correction for bleaching was necessary. $\Delta F$ for calcium traces was equal to $\left(F-F_{0}\right) / F_{0} \times 100$, where $F_{0}$ equals the average $F$ within the first $3 \mathrm{~s}$ of recording. For statistical quantification $\Delta F$ was computed as $\left(F_{1}-F_{0}\right) / F_{0} \times 100$, where $F_{0}$ is the average $F$ over $10 \mathrm{~s}$ before odour switching and $F_{1}$ is the average over $10 \mathrm{~s}$ after odour switching. All imaging strains performed normally in chemotaxis assays.

A two-tailed unpaired $t$-test was used to analyse the data. Where $>1$ comparison was made, a Bonferroni $t$-test was used instead.

To image salt responses, single animals were placed in a perfusion chamber (RC-26GLP,Warner Instruments) under a constant flow rate $\left(0.3 \mathrm{ml} \mathrm{min}{ }^{-1}\right)$ of CTX buffer using a perfusion pencil (AutoMate). Outflow was regulated using a peristaltic pump (Econo Pump, Bio-Rad). Upsteps or downsteps in $\mathrm{NaCl}$ 
concentration were delivered using the perfusion pencil, and switch between control and stimulus solutions was done using manually controlled valves. Solutions contained the indicated amount of $\mathrm{NaCl}$ and in addition $1 \mathrm{mM} \mathrm{MgSO}_{4}$, $1 \mathrm{mM} \mathrm{CaCl}_{2}$ and $5 \mathrm{mM} \mathrm{KPO}_{4}$. The stimulus was delivered for $10 \mathrm{~s}$ starting on the 10th second from the onset of the movie.

To image olfactory responses, worms were inserted into a microfluidic polydimethylsiloxane chip, a generous gift from $\mathrm{N}$. Chronis, designed to deliver odour stimuli under a fluorescent microscope ${ }^{39}$. Ten seconds after the beginning of each recording, we presented the odour $\left(10^{-4}\right.$ benzaldehyde diluted in S-basal lacking cholesterol) and continued imaging for an additional $30 \mathrm{~s}$. We kept the odour channel open for $5 \mathrm{~min}$ and then resumed imaging. After an additional $10 \mathrm{~s}$, we switched the odour off and completed the recording following an additional $30 \mathrm{~s}$. We used buffer instead of fluorescein in the chip to enhance the signal to noise ratio and eliminate switching artifacts, and unlike previous reports ${ }^{25,26}$ we did not pre-immerse the worms in buffer before each recording.

\section{References}

1. Mukherji, S. \& van Oudenaarden, A. Synthetic biology: understanding biological design from synthetic circuits. Nat. Rev. Genet. 10, 859-871 (2009).

2. Khalil, A. S. \& Collins, J. J. Synthetic biology: applications come of age. Nat. Rev. Genet. 11, 367-379 (2010)

3. Bashor, C. J., Horwitz, A. A., Peisajovich, S. G. \& Lim, W. A. Rewiring cells: synthetic biology as a tool to interrogate the organizational principles of living systems. Annu. Rev. Biophys. 39, 515-537 (2010).

4. Miesenbock, G. \& Kevrekidis, I. G. Optical imaging and control of genetically designated neurons in functioning circuits. Annu. Rev. Neurosci. 28, 533-563 (2005).

5. Deisseroth, K. Optogenetics. Nat. Methods 8, 26-29 (2011).

6. Takamori, S. et al. Molecular anatomy of a trafficking organelle. Cell 127, 831-846 (2006)

7. Bayes, A. \& Grant, S. G. Neuroproteomics: understanding the molecular organization and complexity of the brain. Nat. Rev. Neurosci. 10, 635-646 (2009)

8. McCracken, C. B. \& Roberts, D. C. Neuronal gap junctions: expression, function, and implications for behavior. Int. Rev. Neurobiol. 73, 125-151 (2006).

9. Kelmanson, I. V. et al. Altering electrical connections in the nervous system of the pteropod mollusc Clione limacina by neuronal injections of gap junction mRNA. Eur. J. Neurosci. 16, 2475-2476 (2002).

10. Herve, J. C., Phelan, P., Bruzzone, R. \& White, T. W. Connexins, innexins and pannexins: bridging the communication gap. Biochim. Biophys. Acta 1719, 3-5 (2005).

11. Phelan, P. Innexins: members of an evolutionarily conserved family of gap-junction proteins. Biochim. Biophys. Acta 1711, 225-245 (2005).

12. Scemes, E., Spray, D. C. \& Meda, P. Connexins, pannexins, innexins: novel roles of 'hemi-channels'. Pflugers Arch.: Eur. J. Physiol. 457, 1207-1226 (2009).

13. Rackauskas, M. et al. Gating properties of heterotypic gap junction channels formed of connexins 40, 43, and 45. Biophys. J. 92, 1952-1965 (2007).

14. Starich, T. A., Xu, J., Skerrett, I. M., Nicholson, B. J. \& Shaw, J. E. Interactions between innexins UNC-7 and UNC-9 mediate electrical synapse specificity in the Caenorhabditis elegans locomotory nervous system. Neural. Dev. 4, 16 (2009).

15. Epstein, M. L. \& Gilula, N. B. A study of communication specificity between cells in culture. J. Cell Biol. 75, 769-787 (1977).

16. Pierce-Shimomura, J. T., Morse, T. M. \& Lockery, S. R. The fundamental role of pirouettes in Caenorhabditis elegans chemotaxis. J. Neurosci. 19, 9557-9569 (1999).

17. Bargmann, C. I. \& Horvitz, H. R. Chemosensory neurons with overlapping functions direct chemotaxis to multiple chemicals in C. elegans. Neuron 7, 729-742 (1991).

18. Suzuki, H. et al. Functional asymmetry in Caenorhabditis elegans taste neurons and its computational role in chemotaxis. Nature 454, 114-117 (2008).

19. White, J., Southgate, E., Thomson, J. \& Brenner, S. The structure of the nervous system of the nematode Caenorhabditis elegans. Phil. Trans. R. Soc. Lond. (Biol.) 314, 1-340 (1986).

20. Varshney, L. R., Chen, B. L., Paniagua, E., Hall, D. H. \& Chklovskii, D. B. Structural properties of the Caenorhabditis elegans neuronal network. PLoS Comput. Biol. 7, e1001066 (2011).

21. $\mathrm{Xu}, \mathrm{M}$. et al. Computer assisted assembly of connectomes from electron micrographs: application to Caenorhabditis elegans. PloS ONE 8, e54050 (2013)

22. WormWiring < http://wormwiring.org/> (2014).

23. Redemann, S. et al. Codon adaptation-based control of protein expression in C. elegans. Nat. Methods 8, 250-252 (2011).

24. Srinivas, M. et al. Functional properties of channels formed by the neuronal gap junction protein connexin36. J. Neurosci. 19, 9848-9855 (1999).
25. Chalasani, S. H. et al. Dissecting a circuit for olfactory behaviour in Caenorhabditis elegans. Nature 450, 63-70 (2007).

26. Tsunozaki, M., Chalasani, S. H. \& Bargmann, C. I. A behavioral switch: cGMP and PKC signaling in olfactory neurons reverses odor preference in C. elegans. Neuron 59, 959-971 (2008).

27. Tsalik, E. L. \& Hobert, O. Functional mapping of neurons that control locomotory behavior in Caenorhabditis elegans. J. Neurobiol. 56, 178-197 (2003).

28. Kocabas, A., Shen, C. H., Guo, Z. V. \& Ramanathan, S. Controlling interneuron activity in Caenorhabditis elegans to evoke chemotactic behaviour. Nature $\mathbf{4 9 0}$, 273-277 (2012)

29. Lesch, B. J., Gehrke, A. R., Bulyk, M. L. \& Bargmann, C. I. Transcriptional regulation and stabilization of left-right neuronal identity in C. elegans. Genes Dev. 23, 345-358 (2009).

30. Miesenbock, G. Optogenetic control of cells and circuits. Annu. Rev. Cell Dev Biol. 27, 731-758 (2011).

31. Schiavo, G. et al. Tetanus and botulinum-B neurotoxins block neurotransmitter release by proteolytic cleavage of synaptobrevin. Nature 359, 832-835 (1992),

32. van der Bliek, A. M. \& Meyerowitz, E. M. Dynamin-like protein encoded by the Drosophila shibire gene associated with vesicular traffic. Nature 351, 411-414 (1991).

33. Rabinowitch, I., Chatzigeorgiou, M. \& Schafer, W. R. A gap junction circuit enhances processing of coincident mechanosensory inputs. Curr. Biol. 23, 963-967 (2013)

34. Wenick, A. S. \& Hobert, O. Genomic cis-regulatory architecture and trans-acting regulators of a single interneuron-specific gene battery in $C$. elegans. Dev. Cell 6, 757-770 (2004).

35. Yu, S., Avery, L., Baude, E. \& Garbers, D. L. Guanylyl cyclase expression in specific sensory neurons: a new family of chemosensory receptors. Proc. Natl Acad. Sci. USA 94, 3384-3387 (1997).

36. Tsalik, E. L. et al. LIM homeobox gene-dependent expression of biogenic amine receptors in restricted regions of the C. elegans nervous system. Dev. Biol. 263, 81-102 (2003).

37. Pocock, R. \& Hobert, O. Hypoxia activates a latent circuit for processing gustatory information in C. elegans. Nat. Neurosci. 13, 610-614 (2010).

38. Bargmann, C. I., Hartwieg, E. \& Horvitz, H. R. Odorant-selective genes and neurons mediate olfaction in C. elegans. Cell 74, 515-527 (1993).

39. Chronis, N., Zimmer, M. \& Bargmann, C. I. Microfluidics for in vivo imaging of neuronal and behavioral activity in Caenorhabditis elegans. Nat. Methods $\mathbf{4}$ 727-731 (2007).

\section{Acknowledgements}

We thank C.I. Bargmann for strains, N. Chronis for the microfluidic device and for help in setting it up, Y. Tanizawa and K.E. Busch for reagents, H. Bringmann for help with the codon optimizing, and J. Bai, R.S. Branicky, A.E.X. Brown, M. Ezcurra and R. Straussman for helpful discussions and comments on the manuscript. This work was supported by the MRC grant MC-A022-5PB91. I.R. was also supported by postdoctoral fellowships granted by the Israeli Science Foundation Bikura program, the Edmond and Lily Safra Center for Brain Sciences (ELSC), and the Institute for Medical Research Israel-Canada (IMRIC)

\section{Author contributions}

I.R., M.C. and B.Z. carried out the experiments and analysed the data. All the authors planned experiments and interpreted results. M.T. and W.R.S. supervised the research. I.R. and W.R.S. wrote the paper.

\section{Additional information}

Supplementary Information accompanies this paper at http://www.nature.com/ naturecommunications

Competing financial interests: The authors declare no competing financial interests.

Reprints and permission information is available online at http://npg.nature.com/ reprintsandpermissions/

How to cite this article: Rabinowitch, I. et al. Rewiring neural circuits by the insertion of ectopic electrical synapses in transgenic C. elegans. Nat. Commun. 5:4442 doi: $10.1038 /$ ncomms5442 (2014).

This work is licensed under a Creative Commons Attribution 4.0 International License. The images or other third party material in this article are included in the article's Creative Commons license, unless indicated otherwise in the credit line; if the material is not included under the Creative Commons license, users will need to obtain permission from the license holder to reproduce the material To view a copy of this license, visit http://creativecommons.org/licenses/by/4.0/ 